\title{
Proton pump inhibitors: are they overutilised in $e c$ Chiticr clinical practice and do they pose significant risk?
}

\author{
J. J. Heidelbaugh, ${ }^{1}$ D. C. Metz, ${ }^{2}$ Y.-X. Yang, ${ }^{3}$
}

${ }^{1}$ Department of Family Medicine, University of Michigan, Ann Arbor, MI, USA ${ }^{2}$ Division of Gastroenterology, GI Physiology Laboratory, Acid Peptic Disorders Specialty Program, Digestive and Liver Center, Perelman School of Medicine, University of Pennsylvania, University of Pennsylvania Hospital,

Philadelphia, PA, USA

${ }^{3}$ Division of Gastroenterology

and Center for Clinical

Epidemiology and Biostatistics,

Perelman School of Medicine, University of Pennsylvania,

Philadelphia, PA, USA

Correspondence to: Joel J. Heidelbaugh, MD, FAAFP, FACG Ypsilanti Health Center 200 Arnet Suite 200 Ypsilanti, MI 48198, USA Tel.: +(734) 5443210

Fax: +(734) 4847292

Email: jheide|@umich.edu

\section{Disclosures}

Heidelbaugh and Yang have no conflicts of interest. Metz is a consultant for AstraZeneca and Takeda.

\begin{abstract}
SUM MARY
Proton pump inhibitors are highly effective acid suppressants with decades of use highlighting positive outcomes in millions of patients worldwide, and they offer minimal risk of adverse events. PPIs are considered overutilised when prescribed without an appropriate indication, when patients are left on them 'indefinitely' without appropriate indications and when they are continued after being utilised for most cases of hospital SUP. While several adverse outcomes have been linked to PPI therapy, most data are from retrospective observational studies that may be subject to confounding and bias.
\end{abstract}

\section{Introduction}

Proton pump inhibitors (PPIs) are the most widely used agents for suppression of gastric acid in patients with non-erosive gastroesophageal reflux disease (GERD) and erosive esophagitis, accounting for over $\$ 11$ billion in US sales alone between brand name and generic prescription formulations, whereas approximately $80 \%$ of PPIs worldwide are now purchased over-the-counter (1). Hundreds of clinical trials studying PPIs summarised in the American Gastroenterological Association and the American College of Gastroenterology guidelines have demonstrated superior clinical efficacy and safety in comparison with histamine-2 receptor antagonists (H2RAs) $(2,3)$. Since omeprazole was first released in the early 1980s, there have been millions of favourable patient-years worth of experience with their use. However, as all pharmacological agents (including PPIs) have a risk of potential adverse effects, physicians must weigh potential risks of long-term maintenance against therapeutic benefit. Metz and Yang have summarised that the side effect profile of PPIs includes rare idiosyncratic reactions (e.g. acute interstitial nephritis) and metabolic interactions (especially hepatic cytochrome P450 effects), as well as predictable pharmacological consequences of hypoor achlorhydria and reflex hypergastrinemia (4).

In recent years, dozens of retrospective epidemiological studies examining a wide variety of potentially associated adverse effects of PPIs have been pub- lished. This review article will first describe the pharmacological consequences of proton pump inhibition, then discuss the trend of overutilisation of PPIs in clinical practice and will conclude with a discussion of potential safety concerns in perspective through a critical examination of the specific data regarding these effects. The goal of this manuscript is to demonstrate that PPIs should not be denied to patients likely to benefit from them, yet the lowest effective maintenance dose should be utilised and such patients should be followed regularly to readdress the need for continued therapy.

\section{Physiology of gastric acid secretion}

Gastric acid is produced by both resting and mealstimulated parietal cells, following neurocrine, paracrine, or endocrine stimulation by ligands, such as acetylcholine, histamine or gastrin respectively, which bind to their specific receptors on the basolateral surface of the cell $(4,5)$ (Figure 1). In turn, intracellular second messenger systems are activated leading to protein kinase formation and activation of $\mathrm{H}+/ \mathrm{K}+-$ ATPase enzymes (proton pumps), which fuse with the secretory canaliculus of the parietal cell resulting in acid production, whereby intracellular hydrogen ions are exchanged for extracellular potassium ions (5). Once acid is produced, the lower luminal $\mathrm{pH}$ activates a feedback mechanism to maintain appropriate homeostatic control of acid secretion. This response is mediated primarily by paracrine release of somato- 


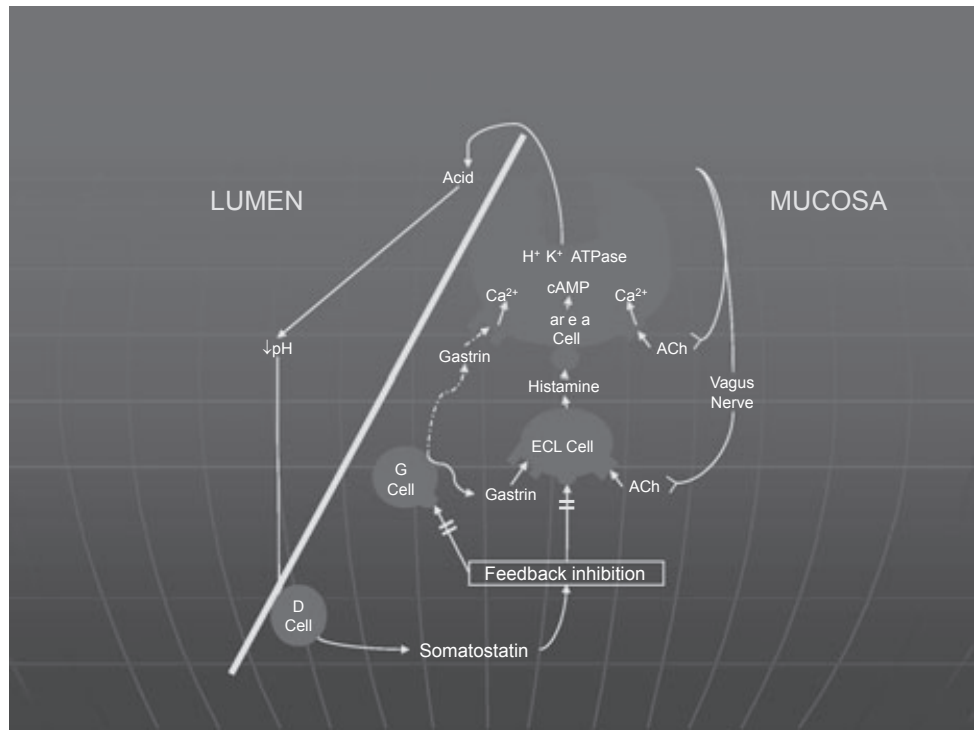

Figure 1 Physiology of gastric acid secretion

statin from gastric antral and corpus D cells, which inhibit G cell production of gastrin and enterochromaffin-like (ECL) cell production of histamine to reverse the stimulus for acid production.

\section{Pharmacology of proton pump inhibition}

Proton pump inhibitors inhibit the final common pathway of gastric acid production, namely activated $\mathrm{H}+/ \mathrm{K}+-\mathrm{ATPase}$ enzymes, preventing the release of hydrogen ions into the gastric lumen $(4,6)$ (Figure 2). They are pro-drugs that are absorbed into the circulation and delivered to the gastric oxyntic mucosa, where they concentrate in the secretory canaliculi of parietal cells, where the $\mathrm{pH}$ is constitutively low. The acidic microenvironment changes the PPI structure allowing them to bind irreversibly with activated $\mathrm{H}+/ \mathrm{K}+$-ATPase enzymes, preventing hydrogen-potassium exchange, leading to an elevation of gastric luminal $\mathrm{pH}$ which subsequently inhibits $\mathrm{D}$ cell release of somatostatin, disinhibiting G cells and ECL-cells in an attempt to restore acid secretory capability $(4,6)$. As PPIs concentrate 1000-fold greater than serum levels in the secretory canaliculi, the resultant $G$ cell release of gastrin and ECL-cell release of histamine impedes activation of additional proton pumps and gastric acid secretion $(4,6)$.

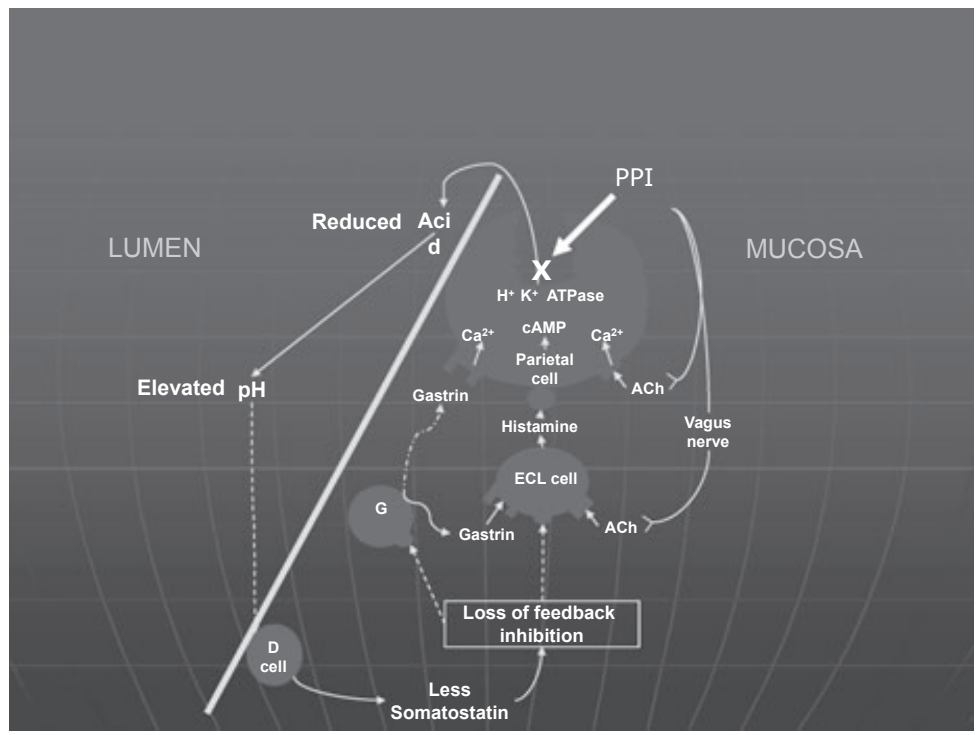

Figure 2 Pharmacology of proton pump inhibition 


\section{Indications for long-term (maintenance) PPI therapy}

Antisecretory agents including PPIs and H2RAs are commonly prescribed or used as short-term, selfdirected, over-the-counter (OTC) therapy for a variety of upper gastrointestinal (UGI) symptoms, without clinically significant pharmacological risks (7). However, these agents are also indicated for longer-term (maintenance) therapy of various acidpeptic conditions including hypersecretory states, such as Zollinger-Ellison syndrome or idiopathic hypersecretion, GERD and non-steroidal anti-inflammatory drug (NSAID) prophylaxis, all of which have potentially severe morbidity and mortality that is significantly ameliorated by PPI treatment (8-11). Stress ulceration is an acid-peptic disorder, almost exclusively seen in severely ill intensive care unit patients that can be mitigated by maintenance antisecretory therapy (AST) during the definable window of risk $(12,13)$. Despite clear indications for long-term PPI therapy, many patients overutilise these medications inappropriately without re-evaluation. Under such conditions, a potential consequence of prolonged PPI therapy is the potential for long-term hypergastrinemia, ECL-cell hyperplasia and parietal cell hypertrophy (4).

\section{Physiological effects of prolonged PPI inhibition}

As PPIs bind directly to enzymes on the secretory canaliculus of the parietal cell (7) rather than to receptors on their basolateral surface (in contrast to
H2RAs), tachyphylaxis does not occur $(4,14)$ (Figure 3). However, concern has been raised for years regarding the potential for rebound gastric acid hypersecretion, following PPI withdrawal (15-17). Studies have demonstrated, symptomatic relapse rates of $50-100 \%$ via an increasing trend in acid secretory capability after PPI exposure $(15,16)$, whereas a systematic review of eight trials evaluating rebound acid hypersecretion after discontinuation of PPIs found that five short-term studies exhibited no evidence for rebound hypersecretion and three longer-term studies of greater than 8 weeks of PPI exposure, demonstrated the presence of rebound only in Helicobacter pylori-negative individuals (17).

Inadomi and colleagues examined the likelihood of withdrawing PPI therapy treatment in patients with UGI symptoms in two studies $(18,19)$. In the first study, the authors attempted to 'step-down' their patients from more than 8 weeks of PPI therapy to H2RA therapy. Sixty per cent of patients were able to step down and the only predictor of failure to withdraw therapy was the presence or absence of heartburn, suggesting that GERD patients were more likely to be PPI-dependent than patients with other foregut symptoms (18). In the second study, the authors examined the potential for stepping down therapy from twice daily to once daily PPI therapy. Step-down was successful in nearly $80 \%$ of this patient cohort, and the one predictor of success or failure to step down to once daily drug was the duration of the prior PPI exposure (19). These data support the overall message of this manuscript in that clinicians should strive to use the lowest effective maintenance PPI dose.

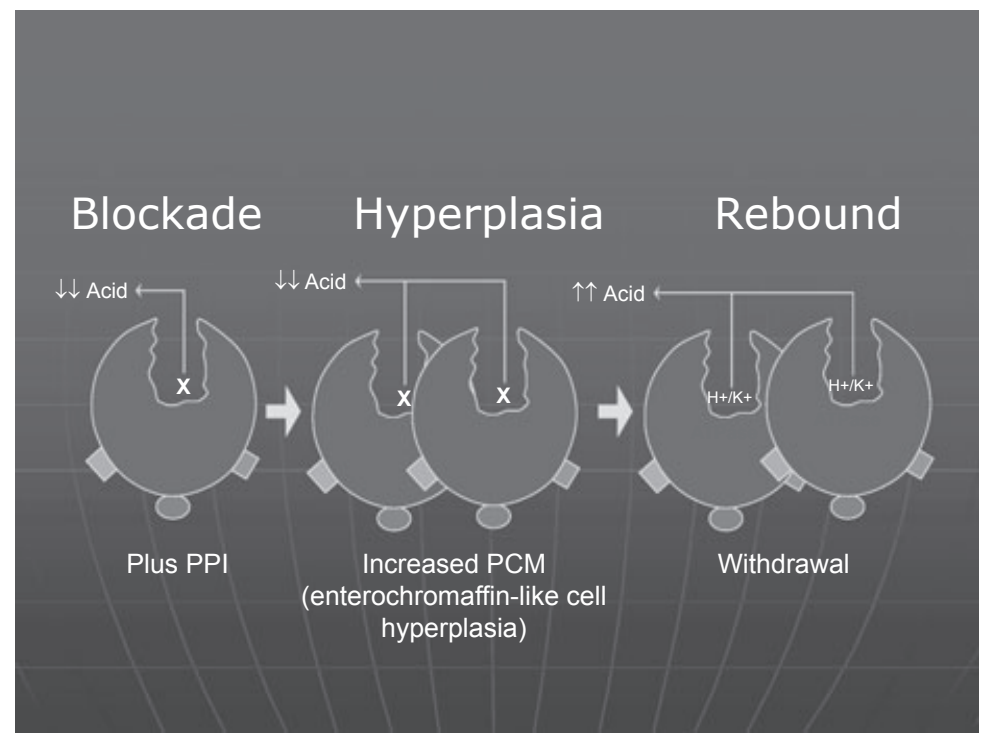

Figure 3 Pharmacological effects of prolonged PPI inhibition 
Recently, two trials in normal subjects examined the potential for PPI withdrawal-induced rebound gastric acid hypersecretion (20,21). The first study was a double-blind, randomised, controlled trial of 120 normal volunteers given 8 weeks of esomeprazole, followed by 4 weeks of placebo vs. 12 weeks of placebo. Mean Gastrointestinal Symptom Rating Scale (GSRS) scores were similar in both groups at baseline, but rebounded after PPI withdrawal reaching statistical significance over weeks $10-12$ as compared with individuals who received placebo for the duration of the study (20). The presence of dyspepsia, heartburn or acid regurgitation was statistically more frequent in the PPI withdrawal group over the same period, and serum gastrin and chromogranin A levels increased in the PPI group and then trended down after withdrawal (20). A second study (21) with similar design yielded similar results, although the symptom rebound occurred sooner after PPI withdrawal (by the 9th week) than in the previous study. The latter study also examined meal-stimulated gastrin levels and found that the area under the gastrin curve increased significantly following PPI therapy, but reverted to normal within a few weeks of withdrawal (21).

To date, only one trial has examined the potential for symptom rebound after PPI withdrawal in GERD patients (22). Metz and colleagues conducted a post hoc analysis of symptom recurrence and gastrin trends in $H$. pylori-negative dexlansoprazole- or lansoprazole-exposed erosive esophagitis patients, who rolled over into the placebo arms of the two doubleblind randomised, controlled dexlansoprazole maintenance registration trials, after healing over 4 or 8 weeks $(23,24)$. This study is of interest because dexlansoprazole may be more potent and has a longer duration of action compared with other PPIs. In this study of approximately 250 patients in each arm there was no evidence for symptom rebound (defined as more severe symptoms during follow-up as compared with prior to diagnosis of erosive esophagitis) or persistent hypergastrinemia in healed erosive esophagitis patients who switched to placebo maintenance therapy (22). Limitations of this study include its post hoc design, the large number of (not unexpected) dropouts during follow-up (because of symptom relapse) and the short duration of PPI exposure (albeit potent).

\section{Reversibility of rebound hypersecretion}

To date, there have been no studies examining whether rebound hypersecretion is reversible and if so, how long it should take to occur. Studies of gastrinoma patients who are cured surgically indicate that it takes approximately 6 months for both basal and maximal acid levels, and antisecretory dose requirements to stabilise postoperatively (25). It should be noted that gastrinoma patients are chronically hypergastrinemic with a mean delay in diagnosis of over 6 years, whereas $H$. pylori-negative PPI recipients exhibit an exaggeration in gastrin response primarily after meals (4).

\section{Overutilisation of PPIs in clinical practice}

The concept of overutilisation of PPIs in clinical practice has received significant attention in recent years, relative to both the potential for adverse side effects and preventable cost-expenditure. Studies spanning over a decade have demonstrated that physicians in the US and UK, in both primary and specialty care, may overprescribe these medications without re-evaluating patients for persistent clinical indication (26-29). Cost-expenditure concerns have led to the development of the step-down therapeutic paradigm (18,19), and subsequent studies have examined the indications for and duration of AST in the outpatient setting (30-32). Similarly, studies have evaluated the practise of stress ulcer prophylaxis (SUP) in the non-intensive care unit (ICU) setting, and have found significant non-judicial and preventable overuse (33-35).

\section{Ambulatory care setting}

Many patients with GERD symptoms often begin with a self-directed trial of OTC AST, whereas most will consult their physician because of persistent symptoms, or to obtain reimbursement for prescribed AST. Physicians often leave patients on PPI therapy indefinitely without readdressing: (i) if the patient takes the PPI daily, (ii) if patient needs to take the PPI daily to prevent symptoms, (iii) if the patient has breakthrough or alarm symptoms suggestive of advanced upper gastrointestinal disease or (iv) if the patient can avoid symptoms without PPI therapy (30).

A retrospective cohort study (30) conducted in a Veterans' Administration hospital evaluated both indications for PPI therapy in the outpatient setting, as well as follow-up parameters and cost-expenditure. Across 946 patients, 35\% were taking PPIs for an appropriate indication, $13 \%$ for symptomatic relief, $19 \%$ for gastroprotection and 33\% had no documented indication for PPI therapy. Appropriate use of PPIs accounted for a mean duration of 1013 days, while inappropriate use, defined as an absence of an appropriate documented indication, accounted for a 
mean duration of 823 days. Nearly $49 \%$ of patients across all four categories received PPIs without re-evaluation, accounting for 1034 patient-years of PPI use. The total cost of inappropriate PPI use was estimated at $\$ 233,994$ based on OTC PPI costs and $\$ 1,566,252$ based on average wholesale price (AWP) costs.

A similar retrospective study (31) conducted on 168,727 managed care patients in Massachusetts found that only $61 \%$ of subjects were taking PPIs for an appropriate upper gastrointestinal diagnosis, including GERD (38\% of total) and dyspepsia (42\% of total). Approximately $39 \%$ of patients lacked appropriate documentation for any UGI diagnosis; almost $50 \%$ had documented symptoms of extraesophageal manifestations of potential UGI disease. Nearly $19 \%$ of subjects had diagnoses or symptoms commensurate with atypical GERD or dyspepsia, whereas there was no subgroup analysis with regard to defined gastroprotection with PPIs. The authors did not assess preventable cost-expenditure in this study.

\section{Stress ulcer prophylaxis}

According to the American Society of Health System Pharmacists (ASHP) guidelines published in 1999 (13), SUP is not indicated in non-ICU patients with fewer than two risk factors for clinically important bleeding (e.g. head or thermal injury, hepatic or renal transplantation, multiple trauma or spinal cord injury, history of gastric ulceration 1 year prior to admission, sepsis, ICU stay of greater than a week, overt or occult bleeding for at least 6 days, or chronic corticosteroid therapy). Despite these recommendations, the evidence for prevention of stress ulceration is poor, and the number needed to treat [NNT] to prevent a single episode in the ICU setting approaches 900 (36). There is no current evidence to posit an NNT in the non-ICU setting.

A retrospective study (33) conducted in a university hospital setting tested the hypothesis that many patients admitted to general medical and family medicine non-ICU services were routinely placed on PPI therapy for SUP, when neither the admitting nor the comorbid diagnoses support their use for either treatment or gastrointestinal prophylaxis. The authors also suspected that a large percentage of patients started on a PPI for SUP at admission were discharged from the hospital on a PPI, both routinely and randomly. The results showed that PPIs were used for SUP in almost 90\% of cases, with 22\% of all admitted patients receiving some form of SUP. These findings were commensurate with previous studies that found a $24-52 \%$ incidence of nonindicated SUP $(34,35)$.
Of 1769 patients in the university-based study (33), there was not a single reported case of stress ulceration. Of the $22 \%$ of patients started on a PPI upon admission, 54\% were discharged home on PPI therapy and none had been re-evaluated within a month to document necessity of therapy. Although the most common admitting diagnoses for patients in this study fell under the category of a GI aetiology, only $15.6 \%$ of patients in this diagnostic category received AST documented as SUP. Rheumatological, renal and cardiac admitting diagnoses were most likely to receive SUP with a PPI. Extrapolated over 1 year, inappropriate PPI use accounted for $\$ 44,096$ in inpatient pharmacy costs and $\$ 67,695$ in outpatient pharmacy costs after discharge, resulting in a total of $\$ 111,791$ in preventable expenditure.

\section{PPI safety concerns}

Safety concerns related to PPIs have centred on the significant physiological changes induced by PPI therapy, and the considerable risks of morbidity and mortality associated with potential adverse effects. A systematic review by Heidelbaugh and colleagues (37), outlines the risk ratios of commonly associated adverse events associated with PPI therapy (Table 1). Despite the increasing attention to this issue, many clinicians believe that the safety concerns associated with PPI therapy, whether real or not, are irrelevant in practise simply because the reported overall risk estimates for many of the adverse effects were modest.

Observational studies are susceptible to bias and confounding. For example, although selection or recall bias is generally not a major concern in population-based studies using collected data, protopathic bias (e.g. medications used to treat early signs of the outcome of interest may appear to be associated with the outcome) could have influenced the reported association between PPI therapy and the risk of community-acquired pneumonia (38). Without randomisation, observational studies cannot account for unmeasured confounders. Information on many potential confounders may not be readily available in retrospective studies using medical records (e.g. physical activity or OTC supplemental vitamin and mineral intake).

Although all published studies on the association between PPI therapy and hip fracture included dementia as well as other potentially confounding comorbidities as covariates, they may not be able to adequately capture the gradation of these conditions to fully account for the confounding effects, which could lead to residual confounding. Thus, positive 


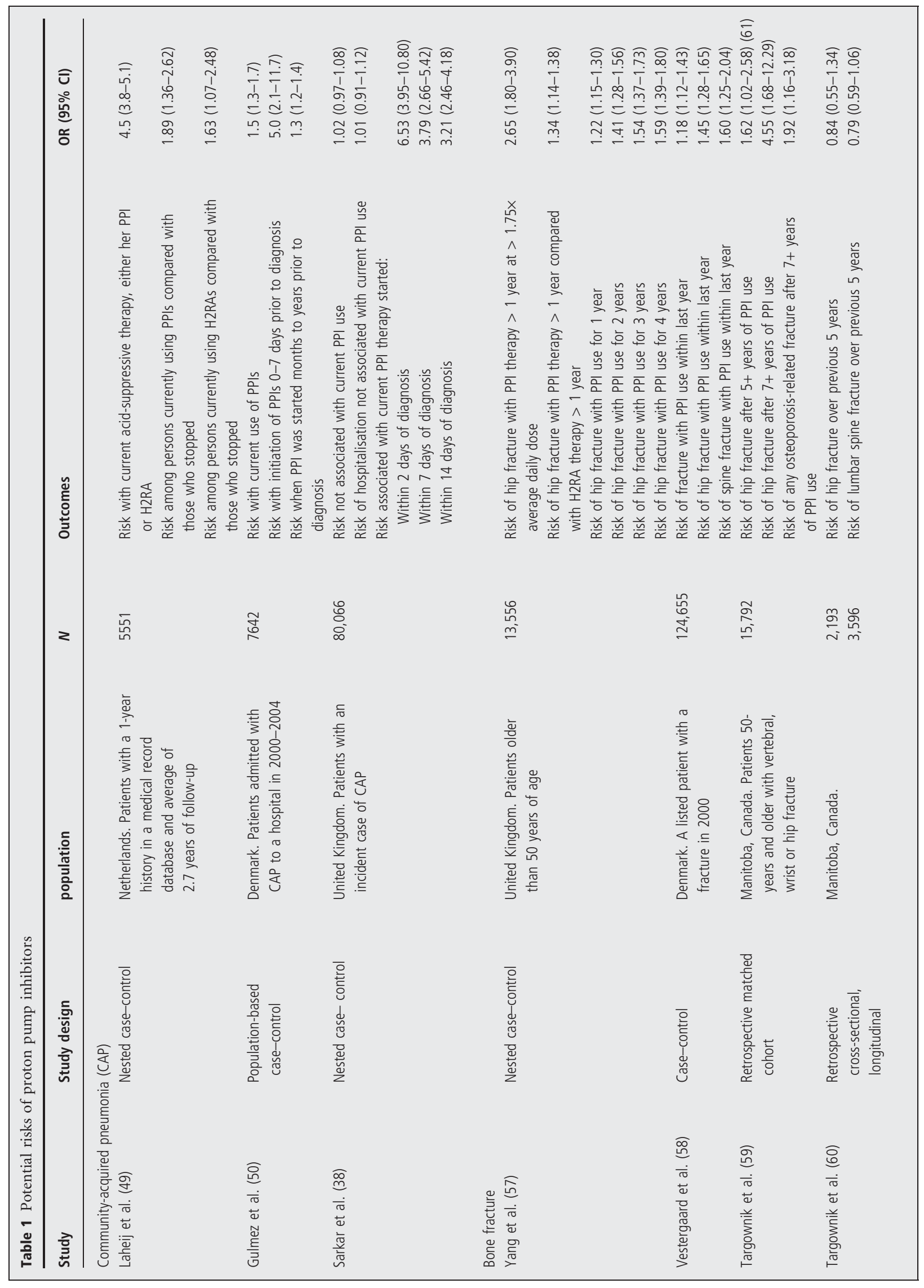




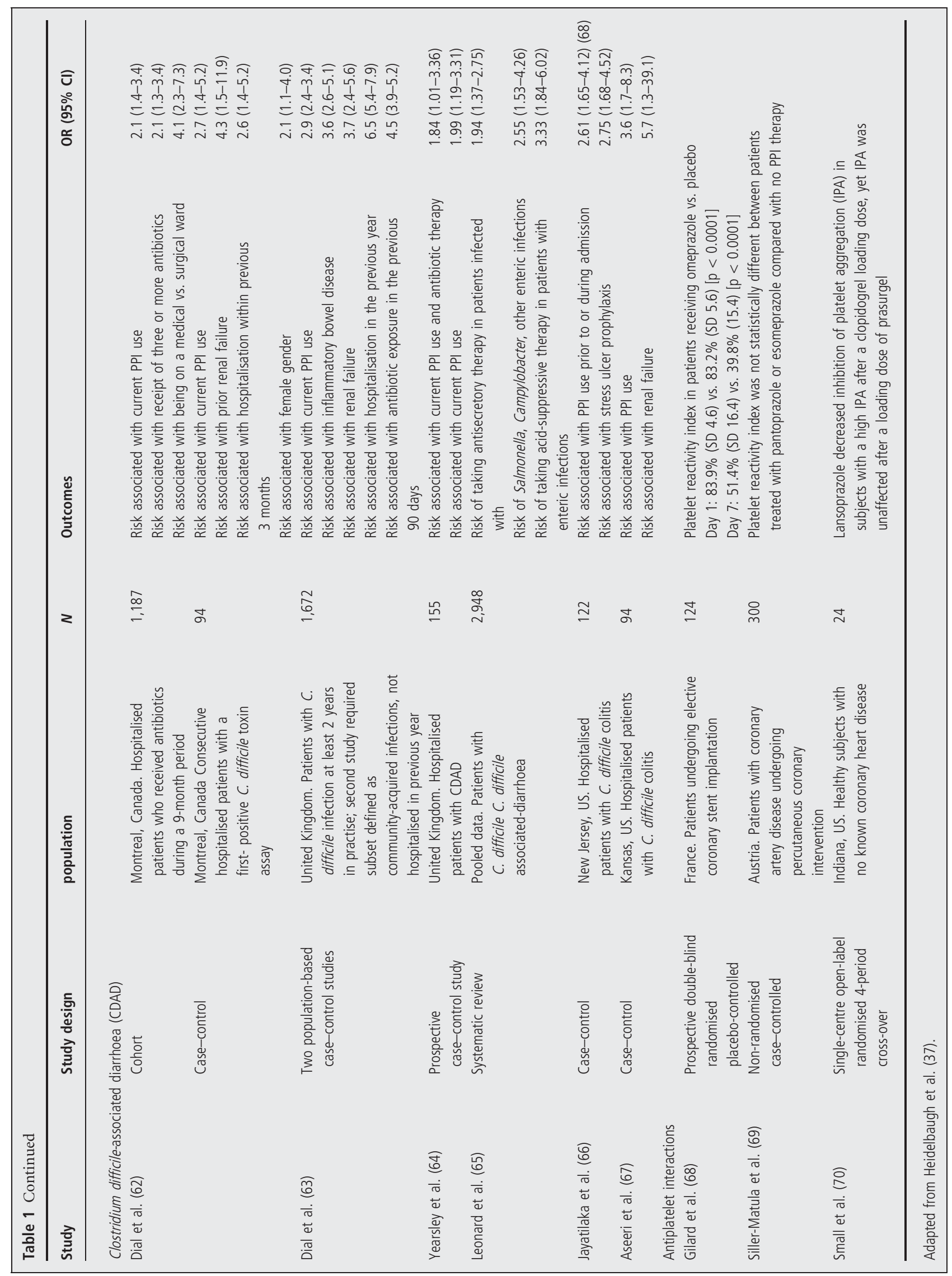


associations observed from these observational studies, particularly when the effect size is relatively modest and the precise underlying mechanisms are incompletely understood, cannot be taken as definitive evidence supporting a causal relationship.

While it is obvious that the reported potential adverse effects associated with PPI therapy are important public health concerns (even at modest to moderate magnitudes), what is unclear yet imperative to consider is whether these potential associations are truly causal. As stated earlier, the strong acid-suppressive effect of PPIs is maintained as long as therapy is continued (39). In addition, the pharmacodynamic interaction of PPIs with CYP2C19 P450 metabolism also appears to be a real phenomenon $(8,40,41)$.

Nearly all potential safety concerns of PPIs fall into three major categories: (i) the direct effect of gastric acid suppression itself (e.g. vitamin B12 deficiency, community-acquired pneumonia, enteric infections including Clostridium difficile-associated diarrhoea [CDAD] and mineral malabsorption, leading to osteoporotic fracture), (ii) the physiological response to the acid suppression (e.g. hypergastrinemia leading to increased cancer risk or hyperparathyroidism and rebound hypersecretion after PPI withdrawal) and (iii) the pharmacodynamic interaction with the metabolism of other medications (e.g. PPI and clopidogrel interaction). Overall, there is sufficient biological plausibility to justify a careful investigation to determine whether these theoretical mechanistic connections translate into clinically important adverse effects.

Almost the entire epidemiological evidence base regarding the safety of PPI therapy to date is composed of retrospective non-randomised trials. Postmarketing surveillance is a critical part of drug safety evaluation, as this mechanism generally consists of spontaneous adverse event reporting systems (e.g. Medwatch) and formal phase IV studies. Spontaneous reporting systems are inexpensive, vital for hypothesis generation, but only rarely are they sufficient for regulatory actions (e.g. PPI-associated hypomagnesemia) (42). Although postmarketing randomised controlled trial (RCT) data to inform safety evaluations are occasionally available [e.g. the COGENT trial for the PPI and clopidogrel interaction (43), de novo dyspepsia after PPI withdrawal $(44,45)]$, observational studies involving large populations using existing medical records with extensive person-years of drug use are the most common type of studies conducted in this setting.

For some safety outcomes, observational studies have shown either consistently no effect with PPI therapy (e.g. colon cancer) (46-48) or highly con- flicting results (e.g. community-acquired pneumonia) $(38,49,50)$. With other PPI safety outcomes (e.g. osteoporotic hip fractures (51-60), CDAD (61-67), interactions with clopidogrel (68-70), although the majority of observational studies have reported an overall positive association, inconsistencies remain regarding the magnitude of risk increase as well as the presence of dose- and/or duration-response.

Recently, a case report highlighted six cases of acute interstitial nephritis (AIN) associated with PPI use, either by temporal association with kidney injury or in response to cessation of PPI therapy (71). The authors admit that the risk of AIN is likely to be very low, a high index of suspicion for potential causality is needed and they hypothesise that if a decline in renal function is observed then PPIs should be stopped.

\section{Future directions}

Unless there are consistently negative observational data [e.g. no observed increased risk of colorectal cancer with short- or long-term PPI use (46)], it is not a sensible approach to simply dismiss the potential risk of PPIs in the setting of suggestive and plausible, yet methodologically limited epidemiological evidence. The rational course of action should be to pursue more definitive evidence through carefully designed clinical research. Future studies should move beyond simply looking at gross epidemiological associations or generating often uninterpretable summary estimates from the invariably heterogeneous pool of observational studies.

Preferred study designs would be either a prospective cohort design to allow better control of potential confounders or, if possible, RCTs. Regarding the issue of PPI-related fracture risk, a recent study from Canada using bone mineral density (BMD) data is a good example of such methodology (60). Although this study was limited by the use of a crude BMD assessment approach and the study cohort being a convenience sample with an unusual risk profile for osteoporosis (4), this study represents an important step in the right direction. A current (non-published) $\mathrm{NIH}$-funded prospective cohort study is comparing the volumetric BMD measured by peripheral quantitative computed tomography (CT), between longterm PPI users and non-users. This type of study can address the primary methodological limitations inherent to the existing observational studies, and ultimately help unravel the nature of the observed epidemiological link between PPI therapy and the adverse effects. If a true causal association can be confirmed, then such studies can also provide useful information regarding potential preventive measures. 


\section{Minimisation of risk from PPIs}

While awaiting more definitive evidence, prescription of PPI therapy in practise can be guided by several general principles to minimise potential risks. First, clinicians should only use PPI therapy in patients who will clearly benefit from it. Importantly, no patients with proper indications for PPI therapy should be deprived of these highly effective medications. It is obvious from the existing evidence that the most consistent and largest increase in the risk of adverse effects is generally associated with long-term and/or high-dose PPI therapy. Therefore, in patients with appropriate indications for PPI therapy, the lowest effective dose should always be prescribed. Unnecessary long-term and/or continuous therapy can be avoided by considering on-demand therapy in suitable patients and conducting periodic review of treatment indications $(18,19)$.

\section{Author contributions}

All authors contributed to the concept and design of this manuscript, literature review, drafts and critical revision and approval of the final manuscript. There was no funding support associated with the development of manuscript.

\section{References}

1 Drug Topics, Pharmacy Facts and Figures. http:// drugtopics.modernmedicine.com/drugtopics/article/ articleList.jsp? categoryId $=7604$ (accessed August 2011)

2 Kahrilas PJ, Shaheen NJ, Vaezi MF et al. American Gastroenterological Association Medical Position Statement on the management of gastroesophageal reflux disease. Gastroenterology 2008; 135: 1383-91.

3 DeVault KR, Castell DO. Updated guidelines for the diagnosis and treatment of gastroesophageal reflux disease. Am J Gastroenterol 2005; 100: 190-200.

4 Metz DC, Yang YX. Safety of proton pump inhibitor exposure. Gastroenterology 2010; 139: 1115-27.

5 Schubert ML, Peura DA. Control of gastric acid secretion in health and disease. Gastroenterology 2008; 134: 1842-60.

6 Sachs G, Shin JM, Howden CW. The clinical pharmacology of proton pump inhibitors. Aliment Pharmacol Ther 2006; 23(suppl): 2-8.

7 Fendrick AM, Shaw M, Schachtel B et al. Self-selection and use patterns of over-the-counter omeprazole for frequent heartburn. Clin Gastroenterol Hepatol 2004; 2: 17-21.

8 Shi S, Klotz U. Proton pump inhibitors: an update of their clinical use and pharmacokinetics. Eur $J$ Clin Pharmacol 2008; 64: 935-51.

9 Metz DC, Strader DB, Orbuch $M$ et al. Use of omeprazole in Zollinger-Ellison syndrome: a prospective nine year study of efficacy and safety. Aliment Pharmacol Ther 1993; 7: 597-610.

10 Kahrilas PJ, Shaheen NJ, Vaezi MF. American Gastroenterological Association Institute technical review on the management of gastroesophageal reflux disease. Gastroenterol 2008; 135: 1392-413.

11 Scheiman JM, Hindley CE. Strategies to optimize treatment with NSAIDs in patients at risk for gastrointestinal and cardiovascular adverse events. Clin Ther 2010; 32: 667-77.

12 Conrad SA, Gabrielli A, Margolis B et al. Randomized, double-blind comparison of immediate-release omeprazole oral suspension versus intravenous cimetidine for the prevention of upper gastrointestinal bleeding in critically ill patients. Crit Care Med 2005; 33: 760-5.

13 American Society of Health-System Pharmacists. ASHP therapeutic guidelines on stress ulcer prophylaxis. Am J Health-Syst Pharm 1999; 56: 347-79.

14 Klinkenberg-Knol EC, Nelis F, Dent J et al. LongTerm Study Group. Long-term omeprazole treatment in resistant gastroesophageal reflux disease: efficacy, safety, and influence on gastric mucosa. Gastroenterology 2000; 118: 661-9.

15 Klinkenberg-Knol EC, Jansen JB, Lamers CB et al. Temporary cessation of long-term maintenance treatment with omeprazole in patients with $\mathrm{H} 2$ receptor-antagonist-resistant reflux oesophagitis. Effects on symptoms, endoscopy, serum gastrin, and gastric acid output. Scand J Gastroenterol 1990; 11: 1144-50.

16 Waldum HL, Arnestad JS, Brenna E et al. Marked increase in gastric acid secretory capacity after omeprazole treatment. Gut 1996; 39: 649-53.

17 Hunfeld NG, Geus WP, Kuipers EJ. Systematic review: rebound acid hypersecretion after therapy with proton pump inhibitors. Aliment Pharmacol Ther 2007; 25: 39-46.

18 Inadomi JM, Jamal R, Murata GH et al. Step-down management of gastroesophageal reflux disease. Gastroenterology 2001; 121: 1095-100.

19 Inadomi JM, McIntyre L, Bernard L et al. Stepdown from multiple- to single-dose proton pump inhibitors (PPIs): a prospective study of patients with heartburn or acid regurgitation completely relieved with PPIs. Am J Gastroenterol 2003; 98: 1940-4.

20 Reimer C, Søndergaard B, Hilsted L et al. Protonpump inhibitor therapy induces acid-related symptoms in healthy volunteers after withdrawal of therapy. Gastroenterology 2009; 137: 80-7.

21 Niklasson A, Lindström L, Simrén M et al. Dyspeptic symptom development after discontinuation of a proton pump inhibitor: a double-blind placebo-controlled trial. Am J Gastroenterol 2010; 105: 1531-7.

22 Metz DC, Pilmer BL, Han C et al. Lack of acid and symptom rebound after withdrawal of 4 to 8 weeks of dexlansoprazole MR or lansoprazole therapy. Am J Gastroenterol 2009; 29: 742-54.

23 Metz DC, Howden CW, Perez MC et al. Dexlansoprazole MR, a proton pump inhibitor with dual delayed-release technology, effectively controls symptoms and prevents relapse in patients with healed erosive oesophagitis. Aliment Pharmacol Ther 2009; 29: 742-54.
24 Howden CW, Larsen LM, Perez MC et al. Clinical trial: efficacy and safety of dexlansoprazole MR 60 and $90 \mathrm{mg}$ in healed erosive oesophagitis - maintenance of healing and symptom relief. Aliment Pharmacol Ther 2009; 30: 895-907.

25 Pisegna JR, Norton JA, Slimak GG et al. Effects of curative gastrinoma resection on gastric secretory function and antisecretory drug requirement in the Zollinger-Ellison syndrome. Gastroenterology 1992; 102: 767-78.

26 Forgacs I, Loganayagam A. Overprescribing proton pump inhibitors is expensive and not evidence based. BMJ 2008; 336: 2-3.

27 Naunton M, Peterson GM, Bleasel MD. Overuse of proton pump inhibitors. J Clin Pharm Ther 2000; 25: 333-40.

28 Batuwitage B, Kingham JCG, Morgan NE et al. Inappropriate prescribing of proton pump inhibitors in primary care. Postgrad Med J 2007; 83: 66-8.

29 Mat Saad AZ, Collins N, Lobo MM et al. Proton pump inhibitors: a survey of prescribing in an Irish general hospital. Int J Clin Pract 2005; 59: 31-4.

30 Heidelbaugh JJ, Goldberg KL, Inadomi JM. Magnitude and economic impact of overutilization of antisecretory therapy in the ambulatory care setting. Am J Manag Care 2010; 16: e228-34.

31 Jacobson BC, Ferris TG, Shea TL et al. Who is using chronic acid suppression therapy and why? Am J Gastroenterol 2003; 98: 51-8.

32 Inadomi JM, Fendrick AM. PPI use in the OTC era: who to treat, with what, and for how long? Clin Gastroenterol Hepatol 2005; 3: 208-15.

33 Heidelbaugh JJ, Inadomi JM. Magnitude and economic impact of inappropriate use of stress ulcer prophylaxis in non-intensive care unit hospitalized patients. Am J Gastroenterol 2006; 101: 2200-5.

34 Nardino RJ, Vender RJ, Herbert PN. Overuse of acid-suppressive therapy in hospitalized patients. Am J Gastroenterol 2000; 95: 3118-22.

35 Zink DA, Pohlman M, Barnes M, Cannon ME. Long-term use of acid suppression started inappropriately during hospitalization. Aliment Pharmacol Ther 2005; 21: 1203-9.

36 Cash BD. Evidence-based medicine as it applies to acid suppression in the hospitalized patient. Crit Care Med 2002; 30(Suppl. 6): S373-8.

37 Heidelbaugh JJ, Goldberg KL, Inadomi JM. Adverse risks associated with proton pump inhibitors: a 
systematic review. Gastroenterol Hepatol 2009; 5: 725-34.

38 Sarkar M, Hennessy S, Yang Y-X. Proton-pump inhibitor use and the risk for community-acquired pneumonia. Ann Int Med 2008; 149: 391-8.

39 Yang YX, Metz DC. Peptic ulcer disease: diagnosis and management. In: Al-Kawas F, ed. Gastrointestinal Diseases: An Endoscopic Approach. 2nd edn. USA: SLACK Incorporated, 2002:385-410.

40 Metz D. Proton pump inhibitor therapy: safety issues. In: Howden CW, ed. Advances in Digestive Disease. Bethesda, MD: AGA Institute press, 2007:3-14.

41 Shin JM, Sachs G. Pharmacology of proton pump inhibitors. Curr Gastroenterol Reports 2008; 10: 528-34.

42 FDA Drug Safety Communication: Low Magnesium Levels can be Associated with Long-Term use of Proton Pump Inhibitor Drugs (PPIs), Vol. 2011. 2011. http://www.fda.gov/Drugs/DrugSafety/ucm245011. htm (accessed 28 March 2012)

43 Bhatt DL, Cryer BL, Contant CF et al. Clopidogrel with or without omeprazole in coronary artery disease. N Engl J Med 2010; 363: 1909-17.

44 Gillen D, Wirz AA, Ardill JE et al. Rebound hypersecretion after omeprazole and its relation to on-treatment acid suppression and Helicobacter pylori status. Gastroenterology 1999; 116: 239-47.

45 Gillen D, Wirz AA, McColl KEL. Helicobacter pylori eradication releases prolonged increased acid secretion following omeprazole treatment. [Erratum appears in Gastroenterol 2004;127(2):694]. Gastroenterology 2004;126:980-8.

46 Yang YX, Hennessy S, Propert K et al. Chronic proton pump inhibitor therapy and the risk of colorectal cancer. Gastroenterology 2007; 133: 748-54.

47 Van Soest EM, Van Rossum LGM, Dieleman JP et al. Proton pump inhibitors and the risk of colorectal cancer. Am J Gastroenterol 2008; 103: 966-73.

48 Robertson DJ, Larsson H, Friis S et al. Proton pump inhibitor use and risk of colorectal cancer: a population-based, case-control study. Gastroenterology 2007; 133: 755-60.

49 Laheij RJF, Sturkenboom MCJM, Hassing R-J et al. Risk of community-acquired pneumonia and use of gastric acid-suppressive drugs. JAMA 2004; 292: 1955-60.
50 Gulmez SE, Holm A, Frederiksen $\mathrm{H}$ et al. Use of proton pump inhibitors and the risk of community-acquired pneumonia: a population-based casecontrol study. Arch Int Med 2007; 167: 950-5.

51 Chiu HF, Huang YW, Chang CC, Yang CY. Use of proton pump inhibitors increased the risk of hip fracture: a population-based case-control study. Pharmacoepidemiol Drug Saf 2010; 19: 1131-6.

52 Pouwels S, Lalmohamed A, Souverein P et al. Use of proton pump inhibitors and risk of hip/femur fracture: a population-based case-control study. Osteoporos Int 2011; 22: 903-10.

53 de VriesF, Cooper AL, Cockle SM et al. Fracture risk in patients receiving acid-suppressant medication alone and in combination with bisphosphonates. Osteoporos Int 2009; 20: 1989-98.

54 Gray SL, LaCroix AZ, Larson J et al. Proton pump inhibitor use, hip fracture, and change in bone mineral density in postmenopausal women: results from the Women's Health Initiative. Arch Intern Med 2011; 170: 765-71.

55 Corley DA, Kubo A, Zhao W et al. Proton pump inhibitors and histamine- 2 receptor antagonists are associated with hip fractures among at-risk patients. Gastroenterology 2010; 139: 93-101.

56 Kaye JA, Jick H. Proton pump inhibitor use and risk of hip fractures in patients without major risk factors. Pharmacotherapy 2008; 28: 951-9.

57 Yang Y-X, Lewis JD, Epstein S et al. Long-term proton pump inhibitor therapy and risk of hip fracture. JAMA 2006; 296: 2947-53.

58 Vestergaard P, Rejnmark L, Mosekilde L. Proton pump inhibitors, histamine $\mathrm{h}(2)$ receptor antagonists, and other antacid medications and the risk of fracture. Calcif Tissue Int 2006; 79: 76-83.

59 Targownik LE, Lix LM, Metge CJ et al. Use of proton pump inhibitors and risk of osteoporosisrelated fractures. CMAJ 2008; 179: 319-26.

60 Targownik LE, Lix LM, Leung S et al. Proton-pump inhibitor use is not associated with osteoporosis or accelerated bone mineral density loss. Gastroenterology 2010; 138: 896-904.

61 Dial S, Alrasadi K, Manoukian C, Huang A, Menzies D. Risk of Clostridium difficile diarrhea among hospital impatients prescribed proton pump inhibitors: cohort and case-control studies. CMAJ 2004; 171: 33-8.
62 Dial S, Delaney JAC, Barkun AN et al. Use of gastric acid-suppressive agents and the risk of community-acquired Clostridium difficile-associated disease. JAMA 2005; 294: 2989-95.

63 Dial S, Delaney JAC, Schneider V et al. Proton pump inhibitor use and risk of communityacquired Clostridium difficile-associated disease defined by prescription for oral vancomycin therapy. CMAJ 2006; 175: 745-8.

64 Yearsley KA, Gilby LJ, Ramadas AV et al. Proton pump inhibitor therapy is a risk factor for Clostridium difficile-associated diarrhea. Aliment Pharmacol Ther 2006; 24: 613-9.

65 Leonard J, Marshall JK, Moayyedi P. Systematic review of the risk of enteric infection in patients taking acid suppression. Am J Gastroenterol 2007; 102: 2047-56.

66 Jayatilaka S, Shakov R, Eddi R, Bakaj G, Baddoura WJ, DeBari VA. Clostridium difficile infection in an urban medical center: five-year analysis of infection rates among adult admissions and association with the use of proton pump inhibitors. Ann Cli Lab Sci 2007; 37: 241-7.

67 Aseeri M, Schroeder T, Kramer J et al. Gastric acid suppression by proton pump inhibitors as a risk factor for Clostridium-difficile-associated diarrhea in hospitalized patients. Am J Gastroenterol 2008; 103 : 2308-13.

68 Gilard M, Arnaud B, Cornily JC et al. Influence of omeprazole on the antiplatelet action of clopidogrel associated with aspirin. The randomized, doubleblind OCLA (Omeprazole CLopidogrel Aspirin) Study. J Am Coll Cardiol 2008; 51: 256-60.

69 Siller-Matula JM, Spiel AO, Lang IM et al. Effects of pantoprazole and esomeprazole on platelet inhibition by clopidogrel. Am Heart J 2009; 157: 148.

70 Small DS, Farid NA, Payne CD et al. Effects of the proton pump inhibitor lansoprazole on the pharmacokinetics and pharmacodynamics of prasurgel and clopidogrel. J Clin Pharmacol 2008; 48: 475-84.

71 Ray S, Delaney M, Muller AF. Proton pump inhibitors and acute interstitial nephritis. BMJ 2010; 341: 668-70.

Paper received September 2011, accepted March 2012 